\section{Clinical Profile and Outcome of COVID- 19 Among Immunocompromised Children}

This retrospective study describes the clinical profile, risk of infection and outcome of coronavirus disease-19 in immunocompromised children. It was found that children on immunosuppressant medication has 2.89 times increased risk of infection $(P=0.01)$. Disease manifestation was asymptomatic $(P=0.01)$ or mild with predominant gastrointestinal symptoms $(P=0.02)$ without alteration in immunosuppressive treatment regime.

Key words: COVID-19, Children, Immunocompromised, Outcome.

Severe acute respiratory syndrome coronavirus 2 (SARS-CoV2) infection in children manifests as mild to moderate disease with low fatality [1]. There is limited information about susceptibility to SARS-CoV-2 infection, clinical profile and outcome among immunocompromised children in India. Published literature has revealed milder disease and favorable outcome without discontinuation of immunosuppressive medication in these children [2-6]. We, herein describe the clinical features and outcome of immunocompromised children diagnosed with SARS-CoV-2 infection at our center.

This was a retrospective analysis of data from 1 June to 31 October, 2020 at a tertiary care center of a teaching hospital. The analysis was approved by institutional ethics committee. All children admitted during the study period were screened for SARS-CoV-2 infection and those who were diagnosed with coronavirus disease 19 (COVID-19) were classified into immunocompromised and immunocompetent. All immunocompromised children in the present study were either on anticancer drugs or immunosuppressive therapy for kidney diseases, and fulfilling criteria for immunocompromised state [6]. The variables extracted from the hospital records were age, gender, presenting symptoms, complications, severity of illness, diagnosis, laboratory investigations, imaging findings, treatment and outcome. The diagnosis of COVID-19 was made by positive reverse transcription polymerase chain reaction (RT-PCR) test for SARS-CoV-2. The severity of illness was classified as per guidelines given by Ministry of Health and Family Welfare, Government of India [7], and children were treated as per unit protocol without alteration in immunosuppressive treatment regime. Proportions were compared using Fisher exact test.

Data of 409 children were extracted during the study period; 162 (396\%) of these were immunocompromised. Of the 409 children, 26 (6.3\%) were diagnosed with SARS-CoV-2. Data of one child was incomplete and he was excluded from the analysis. The proportion of SARS-CoV-2 positivity in immunocompromised and immunocompetent children was $16(9.9 \%)$ and 9 (3.6\%), respectively [OR (95\% CI) 2.89 (1.24-6.73); $P=0.01]$. Comorbidities were present in $184(44.9 \%)$ children, mainly malignancy in $78(18.9 \%)$ and nephrotic syndrome (NS)
Table I Clinical Profile and Outcome of Children with SARSCoV-2 Infection

\begin{tabular}{|c|c|c|}
\hline \multirow[t]{2}{*}{ Variables } & \multicolumn{2}{|c|}{ Children with COVID-19 } \\
\hline & $\begin{array}{l}\text { Immuno- } \\
\text { competent } \\
(n=9)\end{array}$ & $\begin{array}{r}\text { Immuno- } \\
\text { compromised } \\
(n=16)\end{array}$ \\
\hline Comorbidity $^{a}$ & 5 & 16 \\
\hline \multicolumn{3}{|l|}{ Severity of illness } \\
\hline Asymptomatic $^{b}$ & 0 & $8(50)$ \\
\hline Mild & 0 & $3(18.7)$ \\
\hline Moderate & $5(55.5)$ & $3(18.7)$ \\
\hline \multicolumn{3}{|l|}{ Severe $(n=6)$} \\
\hline Septic shock & $4(44.4)$ & $2(12.5)$ \\
\hline ARDS & $2(22.2)$ & $1(5.9)$ \\
\hline \multicolumn{3}{|l|}{ Clinical features } \\
\hline Fever & $9(100)$ & $7(43.7)$ \\
\hline Cough and difficulty in breathing & $8(88.8)$ & $3(18.7)$ \\
\hline Diarrhea and vomiting & 0 & $7(41.1)$ \\
\hline Convulsion and altered sensorium & $3(33.3)$ & 0 \\
\hline Abdominal pain & $2(22.2)$ & $5(29.4)$ \\
\hline \multicolumn{3}{|l|}{ Treatment ${ }^{d}$} \\
\hline Hospitalization & $9(100)$ & $8(50)$ \\
\hline Oxygen & 4 & 2 \\
\hline Vasopressor requirement & 4 & 2 \\
\hline Died & $2(20)$ & $2(12.5)$ \\
\hline
\end{tabular}

Data presented as no. or no. (\%). Immunocompetent:1 each with thalassemia, Wilson disease, diabetes, epilepsy and congenital hydrocephalous with shunt; Immunodeficient: 8 children with nephritic syndrome, 2 with Non-Hodgkin lymphoma and 1 each of Hodgkin lymphoma, acute lymphoblastic leukemia, acute myeloid leukemia, myelodysplastic syndrome, aplastic anemia and retinoblastoma, ${ }^{d}$ Only one immunocompetent patient required ventilation.

in $67(16.3 \%)$. The clinical profile and outcome of children with COVID-19 is shown in Table I. All children with severe COVID-19 disease had features of sepsis and shock; however, 3 children had acute respiratory distress syndrome (ARDS). Only 12 children with COVID-19 underwent blood biochemistry, and reports revealed anemia $(n=6)$, leucopenia $(n=5)$, neutrophil-lymphocyte ratio $>3(n=5)$ and thrombocytopenia in 3 children. High resolution computed tomography of chest showed ground glass opacities in two and air-bronchogram in three children. One child presented with severe abdominal pain and computed tomography of abdomen was suggestive of pancreatitis, he also had elevated amylase $(217 \mu / \mathrm{L})$ and lipase $(365 \mu / \mathrm{L})$.

Immunocompromised children had a significantly higher risk of SARS CoV-2 infection which may be due to the need of frequent hospital visits for their medications (chemotherapy), which exposed them to get infection. However, they had a lower hospitalization rate $[8(50 \%)$ vs $9(100 \%), P=0.01]$ as compared to immunocompetent children possibly because of day care 
treatment protocol. The occurrence of disease was lesser in immunocompromised children, with most being either asymptomatic or with mild disease, and with complete recovery. It is possibly due to weaker immune response under the influence of immunosuppressants. Similar observations were reported in a survey conducted in 25 countries ( $>200$ children tested and 10000 at risk) among children on anticancer treatment [8] and in a systematic review of 16 articles ( 100 adults, 10 children) by Minotti, et al. [5], which concluded that these children had asymptomatic or mild disease, and had a favorable outcome. Severe disease manifestations and require-ment of intensive care, respiratory support, and inotropes were comparable between the two groups. Overall mortality was $16 \%$ and this might be a reflection of high incidence of non-COVID sepsis and associated complications in these patients. In a study on 113 children with kidney diseases receiving immunosuppressive medications from 30 countries; authors found that only $9.7 \%$ had severe grade of disease [3]. Features of relapse, or new organ involvement (pancreas) or new onset glomerulonephritis have been seen in children with nephrotic syndrome in present study and this might be like other viruses, SARS Co-V-2 infection may also precipitate relapses or infect new organ $[9,10]$.

The present study has few limitations, one being retrospective analysis and small sample size, and short term followup. Additionally, admitting policy kept on changing during the study period depending on government guidelines. We conclude that children with immunosuppressant medication are at an increased risk of SARS Co-V-2 infection, and disease manifestations may be asymptomatic or mild with predominantly gastrointestinal symptoms.

Contributors: SKR, RP, VG, OPM: concept, design, drafting of the manuscript, critical analysis; AK: acquisition of data, analysis and interpretation. All authors approved the final version of manuscript, and are accountable for all aspects related to the study.

Funding: None; Competing interests: None stated.

\section{Sunil Kumar RaO, * Ashutosh Kumar, Rajniti Prasad, VineETa Gupta, OM Prakash Mishra Department of Pediatrics, IMS-BHU, Varanasi, Uttar Pradesh \\ *10111drsunilrao21@gmail.com}

\section{REFERENCES}

1. Meena J, Yadav J, Saini L, et al. Clinical features and outcome of SARS-CoV-2 infection in children: A systematic review and metaanalysis. Indian Pediatr. 2020;57:820-26.

2. Marlais M, Wlodkowski T, Vivarelli M, et al. The severity of COVID-19 in children on immunosuppressive medication. Lancet Child Adolesc Health. 2020;4:e17-18.

3. Marlais M, Wlodkowski T, Al-Akash S, et al. COVID-19 in children treated with immunosuppressive medication for kidney disease. Arch Dis Child. 2020 Dec 21; archdischild-2020-320616 (Online ahead of print).

4. El Dannan H, Al Hassani M, Ramsi M. Clinical course of COVID19 among immunocompromised children: A clinical case series BMJ. Case Rep. 2020;13:e237804.

5. Minotti C, Tirelli F, Barbieri E, et al. How is immunosuppressive status affecting children and adults in SARS-CoV-2 infection? A systematic review. J Infection. 2020;81: e61-66.

6. Vasudevan A, Mantan M, Krishnamurthy S, et al. Managing children with renal diseases during the COVID-19 pandemic. Indian Pediatr. 2020;57:641-51.

7. Government of India, Ministry of Health and Family Welfare Guidelines on clinical management of COVID-19. Directorate General of Health Services (EMR Division); p. 3-5. Accessed December 23, 2020. Available from: https://www.mohfw.gov.in/ pdf/ Guidelines on Clinical Management of COVID 1912020.pdf

8. Hrusak O, Kalina T, Wolf J, et al. Flash survey on severe acute respiratory syndrome coronavirus- 2 infections in paediatric patients on anticancer treatment. Euro J Cancer. 2020;132:11-16.

9. Alvarado A, Franceschi G, Resplandor E, et al. COVID-19 associated with onset nephrotic syndrome in a pediatric patient: Coincidence or related conditions? Pediatr Nephrol. 2021;36:205-07.

10. Harambat J, Allard L, Godron-Dubrasquet A. Relapse rate of nephrotic syndrome in the time of COVID-19. Pediatr Nephrol. 2021; 36:211-12.

\section{$\overline{\text { CLIPPINGS }}$}

f

A comparison of post-vaccination hepatitis B surface antibody level on the large and appropriate for gestational age infants (Clin Exp Vaccine Res. 2021;10:47-51)

Hepatitis B infection is the most common cause of chronic hepatitis leading to cirrhosis and carcinoma. Perinatally acquired hepatitis $\mathrm{B}$ infection has the highest rates of progression from acute to chronic disease compared to an infection at later age. Hepatitis $B$ vaccination is important in reducing the global burden of the disease. The researchers enrolled 132 infants aged 7 months, one month after the last (4th) dose of recombinant DNA hepatitis B vaccine containing pentavalent vaccine. All infants were negative for hepatitis B surface antigen and hepatitis B core antibody. On the basis of birthweight, participants were divided into group 1: weighing between 2-4 $\mathrm{kg}(n=63)$, appropriate for gestational age, and group 2: weighing $>4 \mathrm{~kg}(n=69)$, large for gestational age. Anti$\mathrm{Hbs}$ antibody titers $\geq 10 \mathrm{IU} / \mathrm{L}$ were taken as adequate. Mean birthweight of the groups was $2.98(0.53)$ and $4.19(0.19) \mathrm{kg}$, respectively. The mean (SD) anti-HBs antibody titer in group 1 and 2 was $13701.0(11744.4)$ and 8997.1 (2827.2), respectively (95\% CI of difference, -7607.4 to $-1800.2 ; P=0.002$ ), even though the titers were in protective range in both the groups.

RaJesh Kumar Meena raj.mamc@gmail.com 